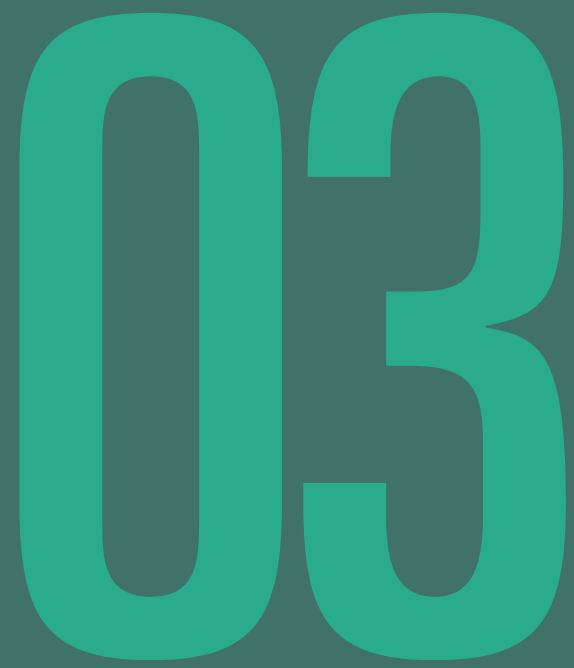

\title{
LA INTERACCIÓN DEL USUARIO CON EL DISEÑO WEB EN FUNCIÓN DE SU COMPOSICIÓN CROMÁTICA, APROXIMACIONES TEÓRICAS.
}

The interaction with the web design based on its chromatic composition, theoretical approaches.

Dra. Martha Judith Soto Flores martha.soto@correo.tij.ibero.mx Universidad IBERO Tijuana

Doctorado en Artes. Universidad de Guanajuato http://orcid.org/0000-0003-2615-879X

Mtra. Ilda Yareli Macias González al150173@correo.tij.ibero.mx Maestría en Diseño Estratégico Digital. Univ. IBERO https://orcid.org/0000-0002-9844-9273

Mtra. Raissa Carolina Lara Corvera 149351@correo.tij.ibero.mx Maestría en Diseño Estratégico Digital. Univ. IBERO https://orcid.org/0000-0002-7976-1579 
Palabras Clave: Color, composición, diseño web, usuario, visión.

Keywords: Colour, composition, web design, user, vision.

\section{Resumen:}

El trabajo aborda la interacción del usuario con el diseño web en función de su composición cromática y al respecto se plantea la realización de una investigación enfocada en el estudio del color, fundamentos básicos de teoría del color, su aplicación e interacción, además de reacciones emotivas que éste pueda generar. Partiendo de lo anterior, se analizan los principios del color aplicados para que un sitio web sea efectivo y funcional, implementando correctamente los colores, no sólo de forma estética sino de forma estratégica, logrando asi un impacto positivo en la percepción del usuario. Se busca además, determinar si hay una correlación entre las reacciones emotivas y las características del color; en otras palabras, si el uso adecuado del color influye en que el usuario permanezca explorando un sitio web, dependiendo del contexto o su combinación con otros colores, así como la consideración de los usuarios finales y sus requerimientos especiales.

\section{Abstract}

This article deals with the interaction between user and web design according to its chromatic composition and in this regard the research focused on the study of color, basic fundamentals of its theory, the use of color and interactions of color, and emotional reactions that it can generate. Based on the above findings, applied color principles will be analyzed so that a website is effective and functional, correctly implementing colors, not only aesthetically but strategically, thus achieving a positive impact on the user's perception. It will also seek to determine whether there is a relationship between emotional reactions and characteristics of colors, if the appropriate use of color influences the user to continue exploring a website, if the perception of color depends on the context or its combination with other colors, as well as our end users and their particular requirements. 
Martha Judith Soto Flores

\section{ANTECEDENTES}

on muchas las causas para que el usuario reaccione ante el diseño de sitios web; sin embargo, enfocaremos nuestro estudio específicamente en la aplicación del color y cómo éste influye en la decisión final del cliente. Existen estudios previos relacionados con el color en diferentes disciplinas que analizan su importancia en relación con el usuario; es así como estudios e investigaciones relacionadas con la evolución, historia, etnografía (particularmente de cultura), neurociencias, psicología del color y fundamentos básicos de la teoría del color, son un antecedente de las necesidades de crear sitios web con una composición cromática estratégica para lograr lo que los diseñadores proponen en un diseño. Como se ha mencionado, el color ha sido objeto de estudio y ha recibido atención constante en diferentes ámbitos, así como diferentes autores han tratado el tema y en particular la interacción del ser humano con la aplicación del color.

El estudio sobre este caso se realizó conforme a los lineamientos del protocolo arsológico para la organización y presentación de investigaciones sobre artes, diseño y temas relacionados, (Jiménez, 2016), con el objetivo principal que el presente trabajo contribuya a la constitución de una base o punto de partida para continuar con el desarrollo de una 
investigación y estudio formal de más largo alcance sobre las composiciones cromáticas y su función en la interacción del usuario con el diseño de sitios web. En el texto se tocan algunos de los autores tradicionales sobre "teoría del color", que suelen comúnmente ser tomados en cuenta durante la formación profesional de artistas y diseñadores, para luego pasar al más moderno estudio científico del color pues, como señala la neurobióloga Margaret Livingstone (2002), en su libro Vision and art, the biology of seeing, sobre lo que se ha dicho respecto al color en el arte, que también aplica en diseño:

Estoy de acuerdo con Shearman en que gran parte de lo que se ha escrito sobre el color en el arte no tiene sentido. Sospecho que esto es cierto al menos en parte porque, hasta hace poco, se sabía muy poco sobre cómo nuestro cerebro procesa la información sobre el color. Para discutir de manera significativa el color en el arte, o cualquier otra cosa, es imprescindible comprender que el color es importante, incluso esencial, en algunas áreas de la percepción visual y completamente irrelevante en otras. Algunos aspectos de la percepción visual, como el reconocimiento de objetos, el reconocimiento facial y, por supuesto, la percepción del color, dependen en gran medida del color y otros elementos de la visión, como la percepción del movimiento, de la profundidad, la segregación de la figura / suelo ,y la percepción de información posicional. Son daltónicos. (p. 46)

En cualquier caso, el escritor y poeta alemán Johann Goethe, en su libro Zur Farbenlehre, centra sus estudios en el color desde la percepción humana, probando así las modificaciones fisiológicas y psicológicas que afectan al ser humano ante la exposición al color y su reacción ante ello. Su obra, sentó las bases de la psicología del color que conocemos en la actualidad, de la cual Lozano (2017) subraya que:

Una mayor apertura del haz de luz, se pierde el espectro; se manifiesta un borde de color rojizo-amarillo y el otro borde de color azul-cyan, con tonos de blanco entre ellos, y el espectro sólo se plantea cuando estos bordes se acercan lo suficiente a la superposición de los colores. (p. 1)

Además, desarrolló un triángulo con los tres colores primarios, rojo, azul y amarillo, y los relacionó con las emociones, siendo así su investigación considerada la piedra angular para la psicología del color que hoy conocemos. 
En cuanto al trabajo de investigación de Eva Heller, en su libro Psicología del Color (2004), afirma que los seres humanos conocemos muchos más sentimientos que colores; es por eso que cada color puede producir muchos efectos distintos, a menudo contradictorios. Así, los colores pueden significar cosas totalmente opuestas, pues un mismo color negro nos puede evocar misterio y sobriedad. Un amarillo puede parecer hiriente y por otro lado alegre, divertido etc. Su efecto está determinado por su contexto y las personas que trabajan con los colores deberían conocer a fondo esos contextos y esos efectos.

Como resultado de sus estudios, es posible concluir que la relación que existe entre los colores y nuestros sentimientos, parecen demostrar que no se combinan de manera accidental, pues sus asociaciones no son meras cuestiones de gusto, sino experiencias universales que están profundamente enraizadas en nuestro lenguaje y en nuestro pensamiento. La relación de los estudios de Heller con la investigación que realizamos es muy notable pues, aunque ejemplifica perfectamente el defecto señalado por Livingstone, no ha dejado de ser uno de entre todos, un tipo de textos base en la formación de artistas y diseñadores.

Incluso autores tan relevantes como Rudolf Arnheim (1985), autor de Arte y Percepción Visual, que tanto y tan bien aporta a la comprensión de los principios de integración de los bordes en configuraciones, falla de manera evidente al hablar de color, pues escribe hace mucho, cuando -como señala Livingstone- poco o nada se sabía sobre la manera en que los ojos y el cerebro procesan la información visual.

Si es verdad que los gatos y los perros no ven los colores ¿qué es lo que se pierden? De una cosa podemos estar seguros: la ausencia de color debe privarles de una dimensión de discriminación de las más eficaces. Será mucho más fácil distinguir y atrapar una pelota que ruede sobre el césped si se la identifica no sólo por su movimiento, forma, textura, y quizás luminosidad, sino también por el color rojo intenso que la distingue de la hierba verde. (p. 363)

Como lo señaló Livingstone, a la detección de la pelota en movimiento, poco o nada contribuye el color; básicamente todo depende del tono o 'luminosidad' que Arnheim casi deja fuera. Sin embargo, este autor sí tuvo razón en que el color rojo de la pelota sobre la hierba nos 
producirá un gran impacto y dará definición a la naturaleza de esa pelota como algo 'atractivo', 'repulsivo' o 'indiferente, como sostiene Jiménez (2019) en su Tratado General de Semiología:

Así, el significado es una función de la vida y, por tanto, la información no tiene significado en sí misma y no significa para los objetos, sólo tiene significado para los sujetos; lo más seguro es que el significado sea una de las primeras y principales adaptaciones evolutivas de los organismos vivos. Dado que su objetivo es preservar su forma -más que conocer o entender el universo- su aparato sensorial-emotivo completo, evolucionó para anticipar consecuencias conducentes o detrimentales a su supervivencia, así como para evitar gastar valiosos recursos lidiando con situaciones sin consecuencias. (p. 52)

Sus estudios confirman que la aplicación de una composición cromática de forma estratégica al desarrollo de sitios web, son una clave importante para el buen funcionamiento de nuestro diseño y para que el usuario se sienta atraído a realizar lo que deseamos o para que simplemente no abandone el portal en los primeros instantes de su visita.

En el área de las neurociencias, los estudios de la ya mencionada Livingstone (2002), nos señala el funcionamiento a nivel neurológico del ojo humano y de cómo su funcionamiento influye en la forma en que percibimos los colores. Explica que la visión no es como una cámara fotográfica que procesa y transmite imágenes, sino más bien como una computadora que interpreta información; procesamiento de información, no transmisión de imágenes, donde en cada etapa de la visión, las neuronas realizan cálculos u operaciones en sus señales de entrada, de modo que el resultado final es información sobre lo que hay en el mundo y cómo actuar sobre él, no sólo una imagen para mirar.

Ante estos estudios, es posible dilucidar que la vista es un proceso muy complejo de extracción de información biológicamente útil, y por tanto, poca o ninguna relación esencial tiene con las cuestiones de gusto, y poco espacio deja para las interpretaciones personales y culturales del color, ya que la visión incluida la del color- cumple con importantes funciones biológicas evolutivas de supervivencia. Pero este detalle es, precisamente, lo que la hace de gran interés para los artistas visuales y 
diseñadores -incluidos los que se enfocan en el diseño para dispositivos electrónicos-; donde los principios biológicos parecen implicar que se pueda jugar con la percepción del usuario.

\section{EL COLOR EN EL DISEÑO WEB}

El color constituye un elemento fundamental en el esquema compositivo del diseño. El diseño como tal es la expresión de las ideas a través los elementos visuales como las formas, su dirección y tamaño. Al diseñar para web debemos tomar en cuenta que la utilización del color será en el formato RGB (red, green, blue), y que, la mezcla de los 3 colores en su $100 \%$ dará como resultado el color blanco mientras que en $0 \%$ será el negro.

Comúnmente, el proceso de consideración de los colores a utilizar iniciaría con la elección del color principal, para después continuar con la búsqueda de aquellos que combinen con él. Se pueden establecer guías de color dependiendo de su familia o gama cromática, las cuales pueden ser consultadas y reproducidas con precisión en pantalla gracias a su código de color. (Wong, 1999)

En los medios impresos, el color se observa mediante pequeños puntos de tinta mientras que en dispositivos como pantallas y televisores se observan los pixeles, que son puntos de luz coloreada activados por electrones. Para reproducir imágenes coloreadas similares a las de la vida real, se realizan tres reproducciones separadas y simultáneas a través de los filtros rojo, verde y azul.

De acuerdo al trabajo de Margaret Livingstone en su libro titulado Vision and Art (2002), se menciona que nuestro sistema visual combina la secuencia de estos puntos de color en tres maneras distintas: cromática, espacial y temporal, razón por la cual nuestro ojo no ve más que sólo pixeles individuales de los tres colores.

También reulta fundamental comprender lo que es un color y cuáles son los principales factores que lo definen y que, de acuerdo con Jiménez (2019): 
Los colores se definen por tres factores o aspectos a los que nosotros denominamos matiz, saturación y tono, aunque cabe señalar que la nomenclatura usada por diversos autores es variada, confusa y hasta contradictoria. También podemos decir que los matices se modulan por su 'temperatura de color' y que no debemos confundir la 'brillantez' de las fuentes de luz con la 'luminosidad' de las superficies de color. Sin importar el nombre que se le da a cada uno de estos factores, podemos decir con certeza que esos son los que nuestro cerebro procesa e interpreta. (p. 102)

Respecto al concepto de Matiz (Hue), Sidelinger (1985) subraya es el estado puro de un color o grupo cromático, determinado por longitudes de onda que distinguen un color de otro. Mientras Jiménez (2017) la define como:

La longitud de onda calculada por nuestro sistema visual en la luz que vemos, siendo que la luz de longitud de onda más larga que podemos ver nos parece roja y según se va acortando la longitud de onda nos parece respectivamente naranja, amarilla, verde, azul y, la más corta que somos capaces de ver, violeta. (p. 71)

El significado que el usuario de la página web va a atribuir al fondo y las figuras, dependerá de su composición cromática en función de la ya mencionada Teoría de las Reacciones Emotivas, en principio, según Jiménez (2017):

El significado de los matices normalmente depende de su proporción, saturación, bordes y otras condiciones. Sin embargo, algunos matices tienen significados más fáciles de identificar. Por ejemplo, el rojo siempre significa intensidad, un tanto de urgencia en la respuesta; es decir, las cosas rojas normalmente son muy buenas o muy malas, con algunas excepciones. Muchos alimentos se vuelven rojos cuando la concentración química de nutrientes está en su punto más alto [...] Los verdes se asocian con la vegetación; si son cálidos -verde amarillos- se asocian con abundancia, buen clima y bienestar general o, si están en proporción exagerada, con lo sofocante de selvas y bosques tupidos. Por otro lado, los verdes azulados se asocian a plantas amargas, venenosas, espinosas, etc. (pp. 38-39) 
Esto quiere decir que, al diseñar, debemos tomar en cuenta los parecidos que la configuración de color elegida guarde con patrones típicos de la naturaleza, pues este parecido va a producir un significado, según esos patrones sean buenos, malos o indiferentes para nuestra supervivencia.

Tono (Valor o brillo). De acuerdo a Livingstone (2002), el sistema visual que nos permite procesar nuestra percepción acerca del movimiento, espacio, posición y profundidad, es el 'Sistema Dónde' que detecta solamente las escalas de gris. Sin embargo, el 'Sistema Qué' también procesa el tono de los colores. En ese sentido Jiménez (2017), enfatiza que:

La luz que nos llega desde la superficie de un objeto, la reflexividad de su superficie hace variar la cantidad de luz que nos llega, lo que el cerebro interpreta como 'luminosidad' o 'tono', de modo que la luz o color del que se trate puede parecernos claro u oscuro, siendo el tono más claro el blanco y el más oscuro el negro, mientras que los tonos intermedios se conceptualizan como una 'escala de grises.' Hay que aclarar que le llamamos tono y no luminosidad, porque no es una dimensión objetiva de la luz, sino percibida en el color y se ve afectada por muchos factores que no tienen nada que ver con la cantidad de luz real que un objeto refleja. Por ejemplo, percibimos un objeto como más claro en medio de un fondo oscuro, y el mismo objeto parece más oscuro en un fondo claro. (pp. 72-73).

Es decir, al elegir el tono de los colores a usar en el diseño web, hay que tomar en cuenta que el cerebro lo interpreta como densidad, peso, distancia, posición o dinámica de las figuras.

Saturación (intensidad). Saturación se refiere a la cantidad de debilidad o fuerza de un matiz en un determinado valor (Sidelinger, 1985). Pueden emplearse otros términos como pureza, intensidad y colorido. Este concepto representa la pureza o intensidad de un color particular. Un color es más brillante en su estado más puro que cuando es mezclado con cualquier otro color o con el gris (Powell, 1984).

Hay numerosas formas de cambiar la intensidad del color, podemos agregar un gris del mismo tono y el color se volverá menos intenso sin hacerse más claro, más oscuro o cambiar de matiz. También se puede mez- 
clar con otros colores: "Mezclando un color con su complementario (el que se encuentra directamente contrario en el círculo del color), podemos también obtener otras variedades de intensidad." (Powell, 1984, p. 23).

Al componer un diseño para internet, debemos tomar muy en cuenta el nivel de saturación, ya que éste puede cambiar drásticamente el significado; por ejemplo, rojos y naranjas desaturados (cafés) parecen cálidos, agradables y tranquilos, pero cuando son muy saturados, parecen apasionados, violentos, etc.

Ideas Sobre el Color. Papachristos, Tselios y Avouris (2005), describen en su estudio que la utilización acertada del color en los sitios web es uno de los factores más importantes al hablar de efectividad y usabilidad, siendo el color más que un elemento indispensable en el sentido estético. El objetivo de su investigación se basó en identificar los colores y sus posibles combinaciones relacionándolos con un valor emocional mediante un proceso metodológico. Los resultados obtenidos fueron categorizados en cuatro niveles de acuerdo a su importancia:

El atributo de diseño con el efecto más fuerte es la luminosidad del color dominante. En segundo lugar, el nivel de luminosidad del color secundario y su tipo (cálido o frío), el número de colores, el contraste entre los matices. En tercer lugar, los dos colores primarios, la paleta de color y el nivel de contraste. Finalmente, la saturación del color dominante y su tipo (cálido o frío). [...] Se han derivado otras relaciones significativas. Por ejemplo, parece preferible usar más de cuatro colores o combinaciones monocromáticas y análogas. Además de esto, parece preferible usar niveles bajos de contraste entre los colores. Los colores fríos se perciben como más adecuados que los cálidos. Y, si nos gustaría adoptar un esquema de color monocromático, es preferible tener un alto contraste en el brillo. Finalmente, con respecto a la saturación, se encontró que la mejor combinación es el color dominante alto saturado, con el color secundario saturado bajo. (p. 1076)

De acuerdo a Kendall (2005), se observa que la utilización del color de manera adecuada permite, no sólo destacar el contraste entre el primer plano y el fondo, sino también resaltar información importante. Así mismo, destaca cinco combinaciones como las más legibles de un texto en primer plano sobre un fondo, siendo de la más legible: 1 . Negro 
sobre amarillo. 2. Verde sobre blanco. 3. Azul sobre blanco. 4. Blanco sobre azul. 5. Amarillo sobre negro. Donde los menos legibles son rojo sobre verde y azul sobre rojo.

Se deben tomar en cuenta también los aspectos culturales y psicológicos del color, así como su valor agregado al diseño. Hablando del diseño de formularios, por ejemplo, se ha demostrado que el uso exagerado del color pudiera distraer a los usuarios, haciendo que se reduzca su desempeño; sin embargo, en algunos otros casos muy específicos, puede facilitar su uso. Una regla útil para la aplicación del color, por tanto, es no usar más de cuatro cuando se trata con usuarios principiantes y no más de siete para los experimentados.

\section{IDENTIFICACIÓN INTERPRETACIÓN}

En cuanto a los tres conceptos descritos como parte del sistema de color más influyente, (Guzmán, 2011) se basa en Munsell (1898), quien creó los tres conceptos anteriores de una manera "racional y ordenada" para describir el color. Inició su sistema con la creación de una esfera de color y lo llamó "equidistancia percibida"; sistema que consta de los tres conceptos ya mencionados, matiz (hue), tono (brillo) y saturación (intensidad).

Cada color posee y tiene esta cualidad y relación lógica con los otros; de esta forma dispone, ordena y especifica los colores, además se muestra su relación, lo que permite elaborar una gama interminable de variaciones cromáticas y tonales. De acuerdo a este sistema están diseñadas las interfaces de software para ilustración, diseño, fotografía, video y todo lo relacionado con el color digital (Guzmán, 2011, p. 30).

Aplicando estos conocimientos de color al diseño web, con un enfoque emotivo, Grieger y Ellis (1977) mencionan que la emoción incluye significativamente al pensamiento y la conducta. Deseamos porque evaluamos algo como "bueno" o "beneficioso" y, como lo deseamos, nos movemos hacia ello, más que alejarnos. La conducta, incluye algún grado de pensamiento y emoción. Al respecto y de acuerdo con Jiménez (2017), la visión a color tiene una función evolutiva específica para los seres humanos y sus parientes cercanos: 
A diferencia de la mayoría de los mamíferos, los seres humanos y otros simios tenemos un segundo sistema visual "a colores" que distingue los matices y la saturación de los colores, además de su tono. Este sistema visual evolucionó mucho más tarde y tiene dos funciones evolutivas principales; identificar objetos sin necesidad de que se muevan y distinguir las caras de diferentes personas, así como su estado emocional y sus intenciones. (p. 34)

Esto nos dice, primero, que el diseño que realizamos, sólo funciona para seres humanos y otros simios, ya que los demás mamíferos no verán el color y, en segundo lugar, que el color va a determinar si el usuario va a identificar o no las figuras que se le presentan, especialmente si esas figuras incluyen expresiones faciales. Pero además, la paleta de color elegida y su articulación, pueden determinar la respuesta emocional del usuario, que puede ser favorable o desfavorable para el fin que se persigue con el diseño. De este modo Jiménez (2017) argumenta que la elección de la temperatura de color, matiz, saturación y tono produce efectos significativos muy importantes en el arte y el diseño; además determinan la reacción emotiva con la que el observador identifica, mucho más que cualquier indicación de tipo verbal o cultural.

Para hacer una correcta selección de color en sitios web, se debe saber con precisión la sensación específica que se desea transmitir al usuario, además de tomar en cuenta la empatía que se busca generar con nuestro diseño. En principio, la paleta de color se debe elegir en base a la Teoría de Reacciones Emotivas. Es decir que, el sentido o significado emotivo de una configuración de, por ejemplo, una página web, será definido para la mayoría de los humanos, en primera instancia, por sus parecidos con las configuraciones típicas de la naturaleza, que han sido buenas, malas o indiferentes para nuestra supervivencia. Mientras que, en segundo lugar, el significado vendrá definido por las convenciones culturales a las que pertenezcan los individuos. En este punto, según Jiménez (2017), enfrentamos un nuevo tipo de semiótica, desligada de los mecanismos naturales de la semiosis y sujeto sólo a cualesquier juego de reglas que tenga la convención.

Así, si elegimos la paleta y composición de color más idónea, se tendrá un resultado positivo atrayendo, pero sobre todo reteniendo, a nuestros usuarios. Una paleta de colores fríos como el azul o el gris, por ejemplo, será comúnmente utilizada para el sector bancario, dado que el 
azul es asociado evolutivamente con seguridad, confianza y tranquilidad, porque el azul corresponde al cielo y agua, limpios y en calma (síntoma de que no hay amenaza climática inminente y si hay abundancia de recursos) y el gris corresponde a las piedras que son permanentes, resistentes, poderosas en su tranquilidad y superficies rígidas y confiables para el desplazamiento y la acción. Independientemente de sus denotaciones o connotaciones culturales, los diferentes aspectos del color codifican significados, dependiendo la configuración de la que forman parte, por ejemplo, el matiz.

En diseño web, se debe tomar en cuenta que dependiendo del contexto, un mismo color puede aplicarse para mostrar diferentes mensajes, porque, de no hacerlo, corremos el riesgo de codificar un mensaje distinto o incluso opuesto al que se pretende. Esto es así porque como explica Jiménez (2017), la visión a color evolucionó como una mera adaptación por la supervivencia en función de la naturaleza química de los objetos, tal como se revela en sus superficies; pero esto hoy se mezcla con las codificaciones convencionales, dependientes de la cultura de cada sujeto.

Por ejemplo, podemos relacionar un color rojo con la marca Coca Cola, y este mismo color puede cambiar su mensaje radicalmente, utilizándolo para la señal de alto, o como señal de peligro. Un color rojo puede utilizarse también en un sitio web para enlaces y botones, siempre y cuando no se utilice de forma excesiva dado su sentido de urgencia. Por otra parte, utilizar un matiz naranja como color principal de un sitio web, podría hablarnos de una marca positiva y divertida cuando está saturado, porque el matiz naranja se relaciona con materiales orgánicos de consecuencias no urgentes, que suelen aportar comodidad y efectos positivos; más dinámicos cuando el color es saturado, como algunas frutas muy nutritivas.

No obstante, la percepción sobre ese mismo color pudiera codificar una marca positiva y tranquila o conservadora, si se utiliza en sus tonos más oscuros y/o desaturados (cafés), porque corresponde evolutivamente a materiales cómodos pero no consumibles, como la tierra o la madera. Sin embargo, en el contexto de una copa mundial de fútbol, por ejemplo, dicho color será asociado por los aficionados a dicho deporte con la selección nacional de Holanda. 
Para aplicar esto al diseño web, sencillamente dependiendo del tipo de portal que se desee diseñar, se utilizan variedades de saturación, según sea el caso; como ejemplo nuevamente el color rojo, en su estado puro con ausencia total de grises, puede utilizarse para un portal con motivos del día de San Valentín o el amor y amistad, mientras que si se desea generar un portal de temas de horror, crimen, etc. se pueden añadir grises para hacerlo parecer más "sucio". Se utiliza la saturación también en diseño web, como elemento estético para retocar fotografías y así generar la sensación de mayor pureza e intensidad en el color de éstas, así como para enfatizar sus características.

En resumen, sobre los significados del tono o luminosidad, este mismo autor nos indica que los tonos claros se asocian con las cosas ligeras, móviles, frescas y cercanas, mientras los oscuros con las cosas pesadas, quietas, cálidas y lejanas (Jiménez, 2017, p. 39).

El contraste tonal entre secciones y elementos es fundamental para la navegación efectiva en el sitio web, mientras la saturación y el contraste de matiz evitarán que el usuario abandone el sitio al volverse tedioso y aburrido. Debemos tomar en cuenta que dependiendo del uso de combinaciones adecuadas de matiz, saturación y tono, podremos comunicar lo que nos proponemos. Por ejemplo, un sitio web con la mezcla de color negro y rojo, se puede utilizar para dar un aspecto masculino al sitio, mientras que la mezcla de blanco y verde puede transmitir la frescura de algún sitio en el que se hace promoción de alimentos saludables y frescos. En cualquier caso el color no se percibe solo o aislado, sino como parte de las figuras o el fondo y que esto va a cambiar su significado.

\section{HERMENÉUTICA}

El uso del color en el diseño de sitios web y su influencia sobre las diferentes reacciones del usuario, basado en las ideas sobre el color que se plantean distintos autores, su aplicación específicamente en el diseño web no ha llegado a conclusiones suficientemente precisas, pues encontramos pocos estudios que nos indiquen de forma directa y concreta cómo utilizar el color exitosamente en la creación de estas plataformas. Sin embargo, podemos anticipar que una aplicación sistemática de los principios de semiología formal -propuestos por Jiménez (2017)- al diseño de sitios web, a través de un futuro estudio de mayor envergadura que el presente, nos permitirá lograr la efectividad y usabilidad que se busca. 
Los detalles de la composición cromática son percibidos siempre como significativos por el cerebro humano; por lo que, al diseñar en web, debemos tomar en cuenta que el sentido del gusto no será el más importante respecto de la efectividad de la página web, sino el aspecto semiológico formal, derivado de la Teoría de Reacciones Emotivas a que se ha aludido antes. Dejando de lado el esquema estructural y el proceso de lectura de una página, la utilización adecuada del color nos permitirá finalmente cumplir con el objetivo para el que fue creada dicha página. Un CTA (Call to Action o llamada de atención) efectivo, por ejemplo, se destaca por el contraste con el resto de los elementos, y generalmente corresponderá a un color cálido -rojo o amarillo- sobre un fondo frío y con nivel de saturación más alto que -y un tono que contraste con- su contexto.

Al finalizar, podemos concluir que, como primer paso, es fundamental el estudio de nuestros usuarios. Se deben considerar las convenciones culturales predominantes entre el público específico o principal a quien va dirigido el diseño. Por otro lado, el análisis de los textos anteriores nos ha permitido comprender que la percepción del color podría variar dependiendo del contexto, la luz ambiental e incluso, el color que se utilice como fondo de pantalla de un dispositivo digital.

\section{CONSIDERACIONES FINALES}

Este trabajo es un acercamiento al estudio del color enfocado en la aplicación del diseño web. El estudio del color ha despertado a lo largo de la historia la atención de investigadores en ciencias como la biología, física y otras disciplinas. Se trata de un factor de la naturaleza tan fascinante como enigmático, que no deja de sorprender, y que aún no se termina de descifrar por completo en la ciencia, sobre todo en los procesos de percepción del color. Sin embargo, autores como Jiménez (2017), nos muestran que, con base en la biología tanto conductual como fisiológica del ser humano, debemos tomar en cuenta las configuraciones típicas de la naturaleza al hacer la elección de la configuración cromática de un sitio de internet o cualquier otro tipo de diseño. Además de que habremos de tomar las denotaciones y connotaciones culturales de dichas configuraciones, respecto del público específico a quien va dirigido el diseño. 
Nos aventuramos a decir, que de este trabajo se puede desprender una comprensión de que el color en las plataformas digitales es más que simplemente elegir -en base al gusto del diseñador, cliente o usuariouna paleta cromática; sino que se trata de predeterminar la experiencia emocional, el comportamiento, el uso y tránsito de los dispositivos electrónicos al definir la composición cromática siguiendo, por lo menos un juego de principios básicos:

Legibilidad: Depende del contraste tonal, la simplicidad y tipicidad de las figuras.

Impacto: Se correlaciona con las configuraciones cromáticas que en la naturaleza connotan urgencia como los patrones de colores cálidos, saturados, en alto contraste, tono medio y con bordes regulares de animales y plantas venenosas, por ejemplo. Siendo las combinaciones más impactantes la de rojo con blanco o la de amarillo con negro.

Dinámica: Donde los colores con matiz cálido, tono claro o alta saturación serán percibidos como dinámicos, así como las gradientes tonales. Mientras los colores en planos tonales, con matiz frío, tono oscuro o baja saturación son percibidos como estables, duros, etc.

Significado: Depende de una combinación entre la Teoría de las Reacciones Emotivas y las convenciones culturales del público a que va dirigido.

\section{BIBLIOGRAFÍA}

Arnheim, R. (1974). Arte y Percepción Visual. Madrid: Alianza.

Grieger, R. y Ellis, A. (1977). Handbook of rational-emotive therapy. New York: Springer.

Guzmán, M. (2011). Teoría práctica del color. Cuenca: Universidad de Cuenca.

Heller, E. (2004). Psicología del color. Barcelona: Gustavo Gili.

Interact. (2005). Lecture Notes in Computer Science, vol 3585. Springer, Berlin, Heidelberg (3 de marzo de 2019).

Jiménez, J. M. (2016). Artelogía, fundamentación de una disciplina Científica para el estudio del arte. (Tesis doctoral). Universidad de Guanajuato, Guanajuato. 
Jiménez, J. M. (2017). Curso de Semiología. Tijuana: Zona Límite.

Jiménez, J. M. (2019). Tratado General de Semiología. Tijuana: Zona Límite.

Livingstone, M. (2002) Vision and art, the biology of seeing. New York: Harry N. Abrams.

Lozano, P. (2017). La teoría del color de Goethe. Recuperado de https://proyectoidis.org/ la-teoria-del-color-de-goethe/

Papachristos, E., Tselios, N., y Avouris, N. (2005). Inferring Relations Between Color and Emotional Dimensions of a Web Site Using Bayesian Networks. En: Costabile M.F., y Paternò F. (Eds.) Human-Computer Interaction-INTERACT.

Powell, W. (1984). The world of color and how to use it. New York.: Walter Foster Publishing, Inc.

Sidelinger, S. (1985). Color Manual. New Jersey: Prentice-Hall.

Wong, W. (1999). Principios del diseño en color. México: Gustavo Gili. 